\title{
Prevalencia de hipotiroidismo y relación con niveles elevados de anticuerpos antiperoxidasa y yoduria en población de 35 y más años en Armenia. 2009-2010
}

\author{
Hypothyroidism prevalence and its relationship to high levels of \\ thyroid peroxidase antibodies and urinary iodine in a population \\ aged 35 and over from Armenia, 2009-2010
}

Ángela L. Londoño, Marta L. Gallego, Adolfo Bayona y Patricia Landázuri

Facultad Ciencias de la Salud. Universidad del Quindío. Armenia, Colombia. angelalilianal@uniquindio. edu.co,mgallego@uniquindio.edu.co,.albayona@uniquindio.edu.co,plandazu@uniquindio.edu.co

Recibido 23 Marzo 2011/Enviado para Modificación 9 Diciembre 2011/Aceptado 24 Diciembre 2011

\section{RESUMEN}

Objetivos Determinar la frecuencia de hipotiroidismo y su relación con anticuerpos antiperoxidasa y yoduria elevada, con la finalidad de realizar recomendaciones a las autoridades sanitarias sobre el consumo de sal yodada y detección temprana de enfermedad tiroidea.

Métodos Participaron 437 personas de la población general de Armenia (Quindío). Se realizaron pruebas ELISA para Tiroxina-L, hormona estimulante de la tiroides, anticuerpos antiperoxidasa y análisis fotocolorimétrico para yoduria.

Resultados La prevalencia de hipotiroidismo fue de 18,5 \%. Los anticuerpos antiperoxidasa fueron positivos en el $28,9 \%$, con prevalencia significativamente más alta entre aquellos con hormona estimulante de la tiroides mayor a $10 \mathrm{uUI} / \mathrm{ml}$ comparados con valores de 5,1 a $10 \mathrm{uUI} / \mathrm{ml}(\mathrm{O} . \mathrm{R} 3,2)$ y en fumadores (O.R 3,4). La Tiroxina-L fue normal en el $98,2 \%$ de participantes con hormona estimulante de la tiroides mayor a $5 \mathrm{uUl} / \mathrm{ml}$ y en el $92 \%$ de aquellos con valores mayores a $10 \mathrm{uUl} / \mathrm{ml}$. El promedio de yoduria fue de 565,1 ; niveles por encima de $300 \mu \mathrm{g} / \mathrm{l}$ se obtuvieron en un $81,8 \%$ de los participantes.

Conclusiones El aumento en la prevalencia de anticuerpos antiperoxidasa positivo a medida que aumentan los valores de hormona estimulante de la tiroides podría evidenciar una elevado riesgo en Armenia de desarrollo de hipotiroidismo de origen autoinmune; a pesar de los elevados niveles de yoduria, no se logró establecer relación con los niveles de anticuerpos antiperoxidasa ni de hormona estimulante de la tiroides.

Palabras Clave: Hipotiroidismo, enfermedades autoinmunes, autoanticuerpos, yoduro peroxidasa, tiroiditis autoinmune, hormonas tiroideas (fuente: DeCS, BIREME). 


\section{ABSTRACT}

Objectives Determining the prevalence of hypothyroidism and its interrelationship with peroxidase antibodies and high urinary iodine levels as a means for devising a set of recommendations for health authorities regarding the consumption of iodised salt and the early detection of thyroid disease.

Methods 437 people in the municipality of Armenia (Quindío) participated in the study. ELISA tests were performed for free thyroxine, thyroid-stimulating hormone and thyroid peroxidase antibodies; a photocolorimetric analysis was carried out to determine urinary iodine levels.

Results Hypothyroidism prevalence was $18.5 \%$. Thyroid peroxidase antibodies were positive in $28.9 \%$ of the study population, with significantly higher prevalence amongst those with levels $>10 \mathrm{mlU} / \mathrm{mL}$ thyroid-stimulating hormone compared to 5.1 to $10 \mathrm{mIU} / \mathrm{mL}$ in those without it (OR 3.2) and smokers (O.R 3,4). Free thyroxine was normal in $98.2 \%$ of participants ( $>5 \mathrm{mlU} / \mathrm{mL}$ thyroid-stimulating hormone levels) and $92 \%$ in those in whom $>10 \mathrm{mlU} / \mathrm{mL}$ thyroid-stimulating hormone levels were found. The average iodine level was 565.1 ; levels above $300 \mu \mathrm{g} / \mathrm{L}$ were obtained in $81.8 \%$ of the participants.

Conclusions Increased positive thyroid peroxidase antibody prevalence with increasing thyroid-stimulating hormone values could demonstrate a high risk of developing autoimmune hypothyroidism in Armenia; despite high iodine levels, a relationship with thyroid peroxidase antibodies or thyroid-stimulating hormone levels could not be established.

Key Words: Hypothyroidism, autoimmune disease, autoantibodies, iodide peroxidase, thyroiditis, autoimmune, thyroid hormone (source: MeSH, NLM).

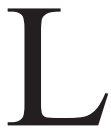

as hormonas tiroideas influyen en la casi totalidad de las funciones del organismo humano (1); los procesos autoinmunes, pueden llevar a sobreproducción o causan destrucción glandular y disminución de la producción hormonal $(2,3)$. La enfermedad tiroidea autoinmune (ETA) constituye la principal causa de hipotiroidismo primario en la edad adulta $(3,4)$.

El hipotiroidismo subclínico $(1,5)$, es de prevalencia alta pero no existe consenso sobre sus consecuencias clínicas o su desarrollo a largo plazo de hipotiroidismo manifiesto. Se asocia con anticuerpos anti tiroideos en un $50 \%$ cuando la TSH (hormona estimulante de la tiroides o thyroid stimulating hormone) se encuentra entre 5 y 10 , y con un $80 \%$ cuando la TSH es mayor a $10(1,6)$.

Existen diversas teorías sobre la patogenia de la ETA; se ha relacionado con la edad avanzada (5), con infecciones provocadas por determinados 
virus o bacterias (3) y en estudios recientes se han encontrado hallazgos repetidamente que asocian los niveles elevados de yodo con el desarrollo de $\operatorname{ETA}(1,8,9,10)$.

Los anticuerpos frente a la peroxidasa tiroidea (anticuerpos antiperoxidasa tiroidea o anti-TPO) antes llamados antimicrosomales, son los más específicos de patología tiroidea; la peroxidasa tiroidea (TPO) es una proteína que se encuentra localizada en la membrana apical del tirocito y es la encargada de la oxidación del yodo tiroideo y favorece la iodinación de los residuos de tirosilo de la tiroglobulina y es el principal antígeno microsomal tiroideo; la presencia de los anti- TPO genera la dishormonogénesis característica de la tiroiditis de Hashimoto; aunque también se encuentran niveles importantes en pacientes con enfermedad de Graves $(1,7)$.

Se plantea entonces el dilema: si la yodización de la sal se está encontrando asociada a más trastornos del funcionamiento tiroideo o realmente protege. No se tienen datos en la región de la frecuencia de ETA ni de hipotiroidismo relacionado con el niveles alterados de yodo; sin embargo entre la comunidad médica, existe la percepción de un aumento en la frecuencia de casos de hipotiroidismo en los últimos años, lo cual justifica iniciar estudios para determinar las causas. Este estudio tuvo como objetivos determinar la prevalencia de anticuerpos antiperoxidasa, de yoduria elevada y su inter- relación con hipotiroidismo con la finalidad de realizar recomendaciones a las autoridades sanitarias sobre el consumo de yodo y la detección temprana de enfermedad tiroidea en población con riesgo así como describir la frecuencia y tipo de manifestaciones clínicas.

\section{MATERIALES Y MÉTODOS}

Población y muestra

Se realizó un estudio de corte transversal; la población de referencia estuvo representada por población general de alto riesgo de 35 y más años, constituida por 437 personas que acudieron voluntariamente a la convocatoria realizada en diferentes sitios de la ciudad de Armenia en los años 2009- 2010. Fueron excluidas del estudio, personas que en el momento del estudio estuvieran en tratamiento con hormonas tiroideas o con historia de hipotiroidismo congénito y cirugía por cáncer de tiroides. 
Los voluntarios firmaron un consentimiento informado y diligenciaron una encuesta para recolección de datos demográficos básicos. El estudio fue aprobado por el comité de Bioética de la Universidad del Quindío.

Muestras. se obtuvo sangre por punción venosa en tubo seco después de doce horas de ayuno, el suero se obtuvo por centrifugación a $2500 \mathrm{~g}$ por 15 minutos, a $4^{\circ} \mathrm{C}$, separado en microtubos. Se tomó una muestra casual de orina en el mismo lapso de tiempo de toma de la muestra sanguínea. Las muestras fueron congeladas a $-20^{\circ} \mathrm{C}$ hasta su uso (dentro de los 20 días siguientes).

Análisis de laboratorio. la yoduria se analizó con el método espectrofotométrico basado en la reacción de Sandell-Kolthoff modificado por Pino y colaboradores (11) y adaptado por nuestro grupo (12); los valores se expresaron en microgramos de yodo por litro $(\mu \mathrm{g} / \mathrm{L})$ y para su interpretación se utilizó la clasificación de la Organización Mundial de la Salud (13): deficiencia severa de yodo $<20 \mu \mathrm{g} / \mathrm{L}$, deficiencia moderada 20-49 $\mu \mathrm{g} / \mathrm{L}$, deficiencia leve 50-99 $\mu \mathrm{g} / \mathrm{L}$, niveles normales $100-199 \mu \mathrm{g} / \mathrm{L}$, riesgo suficiente para hipertiroidismo inducido por yodo $200-299 \mu \mathrm{g} / \mathrm{L}$ y riesgo alto de efectos adversos a la salud (hipertiroidismo autoinmune) $>300 \mu \mathrm{g} / \mathrm{L}$.

La TSH y la T4 L se procesaron con el kit comercial (Monobind/ ACCUBIND), y se leyeron en un equipo de ELISA Stat Fax 303/Plus, en una longitud de onda $450 \mathrm{~nm}$; los valores de referencia normales fueron 0.39-6.19 $\mu \mathrm{UI} / \mathrm{ml}$ para TSH y $0.8-2.0 \mu \mathrm{g} / \mathrm{dL}$ para T4L.

Anticuerpos antiperoxidasa. (Ac-TPO). Se analizaron con el Kit comercial (anti TPO AESKU 7401), se utilizó una dilución de las muestras de suero 1:101. Una vez terminado el ensayo se leyó a $450 \mathrm{~nm}$ dentro de los 30 minutos siguientes en un equipo lector ELISA Stat-fax 303/Plus. Los valores de referencia fueron: rango normal $<40 \mathrm{IU} / \mathrm{ml}$, indeterminado 40 $60 \mathrm{IU} / \mathrm{ml}$, resultados positivos $>60 \mathrm{IU} / \mathrm{ml}$.

\section{Análisis estadístico}

El procesamiento y análisis de la información se realizó en el paquete estadístico SPSS versión 14.0; se realizó un análisis descriptivo y bivariado y se calcularon medidas de asociación estadística no causal, la 
medida de asociación utilizada fue la razón de disparidad (OR) y el test de hipótesis para variables cualitativas fue el chi cuadrado. Para el análisis de variables cuantitativas se determinó la normalidad mediante la prueba de Kolmogorov-Smirnov, para decidir la realización de pruebas paramétricas o no paramétricas. Se exploraron factores sociodemográficos, niveles de yoduria, niveles de Ac TPO buscando asociación estadística a un nivel de 0,05 , se calculó OR con intervalos de confianza.

\section{RESULTADOS}

El estudio incluyó 437 participantes, 4 hombres y 435 mujeres con una media de edad de 52,2 años (IC95\% 49,5-54,8), el 45,7 \% de la población pertenecía a los estratos $1-2,58,6 \%$ al $3-4$ y $2,9 \%$ al estrato 5 ; un 94,4 $\%$ residían en zona urbana. La prevalencia de alteraciones en los niveles de TSH fue de 20,6\% (90/437 participantes); 18,5\% correspondió a hipotiroidismo. De las TSH alteradas se encontraron valores por debajo de $0,39 \mu \mathrm{UI} / \mathrm{ml}$ un $2,1 \%$ (9), se clasificaron como probable hipotiroidismo subclínico valores entre 5,1 - $10 \mu \mathrm{UI} / \mathrm{ml}$ un $62,2 \%$ (56) y por encima de $10,1 \mu \mathrm{UI} / \mathrm{ml}$ un $27,8 \%$ (25) (Tabla 1).

Tabla 1. Niveles alterados de $\mathrm{TSH}^{\mathrm{a}}$ y relación con $\mathrm{T} 4 \mathrm{~L}^{\mathrm{b}}$, Anti- $\mathrm{TPO}^{\mathrm{c}}$ y Yoduria

\begin{tabular}{|c|c|c|c|c|c|c|}
\hline & & & TSH agr & pada & & \\
\hline & & $<0,39$ & $5,1-10$ & $\begin{array}{l}10,1 y> \\
n(\%)\end{array}$ & $5,1 Y>$ & Total \\
\hline TSH & & & & & & \\
\hline № (\%) & $\begin{array}{l}\text { Entre niveles } \\
\text { alterados }\end{array}$ & $\begin{array}{l}9 / 90 \\
(10,0)\end{array}$ & $56 / 90(62,2)$ & $\begin{array}{l}25 / 90 \\
(27,8)\end{array}$ & $\begin{array}{l}81 / 90 \\
(90,0)\end{array}$ & $\begin{array}{l}90 / 90 \\
(100)\end{array}$ \\
\hline & $\begin{array}{l}\text { En total } \\
\text { población }\end{array}$ & $\begin{array}{l}9 / 437 \\
(2,1)\end{array}$ & $56 / 437(12,8)$ & $\begin{array}{l}25 / 437 \\
(5,7)\end{array}$ & $\begin{array}{l}81 / 437 \\
(18,5)\end{array}$ & $\begin{array}{l}90 / 437 \\
(20,6)\end{array}$ \\
\hline T4L & & & & & & \\
\hline № (\%) & $\begin{array}{l}<0,8 \\
0,8-2,0\end{array}$ & $\begin{array}{l}0(0) \\
6(66,7)\end{array}$ & $\begin{array}{l}0(0) \\
55(98,2)\end{array}$ & $\begin{array}{l}2(8) \\
23(92,0)\end{array}$ & $\begin{array}{l}2(2,5) \\
78(96,3)\end{array}$ & $\begin{array}{l}2(2,2) \\
84(93,3)\end{array}$ \\
\hline & $>2$ & $3(33,3)$ & $1(1,8)$ & $0(0)$ & $1(1,2)$ & $4(4,4)$ \\
\hline TPO & & & & & & \\
\hline № (\%) & $\begin{array}{l}<40 \\
40-60\end{array}$ & $\begin{array}{l}4(44,4) \\
1(11,1)\end{array}$ & $\begin{array}{l}43(76,8) \\
2(3,6)\end{array}$ & $\begin{array}{l}14(56,0) \\
0(0)\end{array}$ & $\begin{array}{l}57(70,4) \\
2(2,5)\end{array}$ & $\begin{array}{l}61(67,8) \\
3(3,3)\end{array}$ \\
\hline & $60 y+$ & $4(44,4)$ & $11(19,6)$ & $11(44,0)$ & $22(27,2)$ & $26(28,9)$ \\
\hline Yoduria $^{1}$ & & & & & & \\
\hline № $(\%)$ & $\begin{array}{l}50-99 \\
100-199\end{array}$ & $\begin{array}{l}0(0,0) \\
1(16,7)\end{array}$ & $\begin{array}{l}1(1,9) \\
4(7,5)\end{array}$ & $\begin{array}{l}1(4,2) \\
1(4,2)\end{array}$ & $\begin{array}{l}2(2,6) \\
5(6,5)\end{array}$ & $\begin{array}{l}2(2,4) \\
6(7,2)\end{array}$ \\
\hline & $200-299$ & $1(16,7)$ & $3(5,7)$ & $4(16,7)$ & $7(9,1)$ & $8(9,6)$ \\
\hline & $300 y+$ & $4(66,7)$ & $45(84,9)$ & $18(75,0)$ & $63(81,8)$ & $67(80,7)$ \\
\hline
\end{tabular}


La media de tiroxina libre (T4L) fue de 1,16 $\mu \mathrm{g} / \mathrm{dL}$ (IC95 \% 1,1-1,2); en un 93,3\% los valores fueron normales. El valor promedio de los antiTPO fue de 58,7 IU/ml (IC95\% 37,0- 80,5), con una mediana (Me) de 4,2 (Rango: 0,50- 343,5); en un 67,8\% (n: 61) los valores fueron NEGATIVOS y el 28,9 \% (37) tuvo valores POSITIVOS ( $>$ a 60 ). La prevalencia de anti- TPO positivos entre los pacientes con TSH mayor a 10 fue de $44,0 \%$ y entre aquellos con TSH de 5,1 a 10 fue de un 19,6 \%. (OR 3,2 (IC 95 \% $1,15-8,99 ; p=0,023)$.

El promedio de yoduria fue de 565,1 $\mu \mathrm{g} / \mathrm{L}$ (IC $95 \% 460,6-666,6$ ), la Me 424,2 (R: 61,7- 2538,7 $\mu \mathrm{g} / \mathrm{L}$ ); se encontraron con riesgo excesivo (> $300 \mu \mathrm{g} / \mathrm{L}$ ) un $80,7 \%$ (n: 67). Presentaron yoduria por encima de 300, un $84,9 \%$ de quienes tenían TSH entre 5,1 y $10 \mu \mathrm{UI} / \mathrm{ml}$ y de $75 \%$ entre aquellos con TSH de mayor a 10. No se encontraron relaciones entre los niveles de yoduria y los de anti- TPO (Tabla 1).

Figura 1. Distribución proporcional de la sintomatología referida con resultados de $\mathrm{TSH} \geq$ a $5 \mu \mathrm{Ul} / \mathrm{ml}$

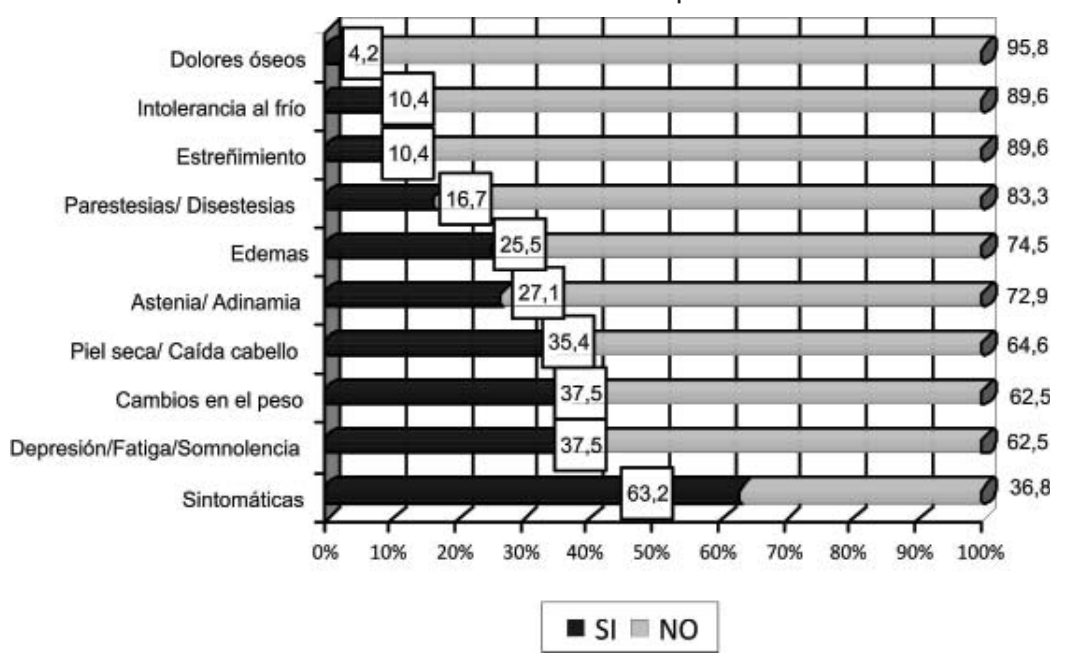

La media de yoduria con niveles de TSH entre 5,1 a $10 \mu \mathrm{UI} / \mathrm{ml}$ fue de $532,8 \mu \mathrm{g} / \mathrm{L}$, mientras que con niveles mayores a 10 fue de $636,4 \mu \mathrm{g} / \mathrm{L}$ $(p=0,83)$. Se estudiaron características del consumo de sal; un 6,5\% adiciona sal a las comidas ya preparadas, un 13,2\% adiciona sal a algunas frutas y $23,7 \%$ consume alimentos salados industriales en paquete. No se encontraron relaciones entre consumo de sal y yoduria. 
La media de edad en pacientes con TSH entre 5,1 y 10 fue de 51,6 años, y en niveles $\geq 10$ fue de 54,4 años (Mann Whitney $p$ 0,52). No hubo correlaciones entre yoduria y edad (Co Pearson $\mathrm{R}=-0,02)$, TPO $(\mathrm{CP} R=$ $-0,2)$ ni TSH (CP R= -0,06).

No se encontraron relaciones entre alteración de hormonas tiroideas, yoduria ni anti-TPO con antecedentes de enfermedades, consumo de medicamentos, medicinas naturales, antecedentes familiares de enfermedad tiroidea, pero el $15,5 \%$ de la población de estudio eran fumadores habituales, encontrando un riesgo significativamente mayor entre los fumadores para presentar anti- TPO positivos (O.R 3,4 IC $95 \%$ 1,02 - 11,6 $p=0,039)$.

Entre los participantes con TSH de 5 y más; un 63,2 \% refirieron alguna sintomatología, la mayoría inespecífica; los síntomas más frecuentemente mencionados fueron: depresión y/o fatiga y/o somnolencia y astenia $\mathrm{y} / \mathrm{o}$ adinamia refirieron un 27, $1 \%$ (Figura 1 ).

No se encontraron diferencias en la sintomatología de acuerdo a la edad $(p=0,52)$, al comparar niveles de TSH de 5,1 a $10 \mathrm{y}>10 \mu \mathrm{UI} / \mathrm{ml}(61,1$ $\%$ vs $68,2 \%, p=0,56)$; tampoco con los niveles de anti $-\mathrm{TPO}<60$ y $\geq 60$ $(67,2 \%$ vs $60,0 \%, p=0,53)$. Entre todos los sintomáticos se encontró que la mayoría $(84,9 \%)$ tuvieron niveles de yoduria por encima de $300 \mathrm{sin}$ diferencia significativa con los asintomáticos $(p=0,147)$.

\section{DISCUSION}

Las disfunciones tiroideas pueden diagnosticarse a través de la realización de TSH y de T3 y T4 libres (14); pero para establecer la etiología de la alteración, se requiere de otras pruebas; para el diagnóstico de ETA se requiere detectar anticuerpos anti tiroideos (14).

La prevalencia de hipotiroidismo aumenta con la edad, y varía con el género y la condición socio-económica; en la población general la prevalencia fluctúa entre $2-8 \%(5,15)$. En los ancianos oscila entre 0,9 y 5,9 $\%$. Las cifras ascienden a 14- 18,2 \% cuando se considera el hipotiroidismo sub clínico (5). En América Latina la prevalencia de hipotiroidismo sub clínico en mujeres post menopáusicas es de 10 a $40 \%$ (6). 
En la ETA la disfunción ocurre por daño tisular y humoral; los antiTPO representan el método más sensible para la detección (16). En nuestro estudio, los anti-TPO positivos (valor de referencia mayor o igual a 60) presentaron una prevalencia de $28,9 \%$, el hallazgo de una prevalencia significativamente más alta de valores positivos entre aquellos con TSH mayor a 10 con relación a quienes tenían valores de 5,1 a 10 (O.R 3,2) podría evidenciar una elevado riesgo en Armenia de desarrollo de hipotiroidismo de origen autoinmune. A pesar de los elevados niveles de yoduria, no se logró establecer relación de esta con los niveles de anti- TPO ni de TSH. En muchos pacientes con función tiroidea normal pueden identificarse anticuerpos antitiroideos (17), sin embargo, se ha descrito que al realizar seguimiento de personas con anti- TPO positivos durante un tiempo, hasta una cuarta parte de ellas pueden llegar a desarrollar hipotiroidismo clínico $(16,17,18)$; por tanto, los anti-TPO podrían ser predictores junto con los cambios en la TSH, de hipotiroidismo (18).

Los anticuerpos anti- TPO son específicos de ETA, pero no de una etiología concreta de la misma. Son positivos en el 80-90 \% de los casos de tiroiditis linfocitaria crónica (Hashimoto), en el 50-80 \% de enfermedad de Graves, en un 20-30 \% de bocios multinodulares y también en el cáncer diferenciado de tiroides (1). Se detectan en un 40-50\% de familiares de un enfermo con patología tiroidea autoinmune. La prevalencia de anticuerpos anti-TPO en estudios poblacionales sin evidencia de enfermedad tiroidea oscila entre el 10-22\%, siendo las tasas 3-4 veces superiores en mujeres; en general, con títulos entre 100 y 400, pero títulos más elevados (400-1.000) sólo se hallan en un $1 \%$ de los individuos de las muestras poblacionales estudiadas (1). Dicha prevalencia es superior en áreas de ingestión adecuada de yodo cuando se compara con poblaciones procedentes de áreas deficitarias de este oligoelemento $(5,6)$; la positividad de anti-TPO se ha correlacionado con disfunción tiroidea, alcanzando una cifra cercana al $50 \%(6)$.

La prevalencia de los anticuerpos anti tiroideos está aumentada en desórdenes autoinmunes; algunas enfermedades que se relacionan con ETA son gastritis autoinmune, vitiligo, miastenia gravis, púrpura trombocitopénica, alopecia, síndrome de Sjögren, enfermedades autoinmunes sistémicas (artritis reumatoide, lupus eritematoso sistémico, esclerosis sistémica progresiva) y enfermedades endocrinas como la diabetes mellitus insulinodependiente $(3,17)$. 
Recientemente se ha investigado la posible inducción de autoinmunidad en infecciones por retrovirus, endógenos o exógenos y se ha encontrado asociación con ciertas especies de Yersinia enterocolítica y retrovirus. Medicamentos asociados a autoinmunidad tiroidea son la amiodarona con una prevalencia entre 1 y $25 \%$; el litio, con una prevalencia entre 7 y 27 $\%$ y el Interferón alfa hasta en un $15 \%$ se asocian con anti- TPO $(3,16,17$, 21,22). Hay investigaciones que apuntan a la influencia de factores genéticos en la patogenia de la enfermedad; se detecta historia familiar de patología autoinmune tiroidea en un $30 \%$ de los casos $(3,17,23)$.

El cigarrillo se ha asociado con tiroiditis de Hashimoto y al parecer podría estar relacionado con la presencia de tiocianatos en el humo del cigarrillo (17). Uno de los hallazgos en este estudio fue precisamente encontrar un riesgo significativamente mayor entre los fumadores para presentar anti- TPO positivos (OR: 3,4 ).

Según los criterios epidemiológicos de la OMS una nutrición de yodo óptima se alcanza cuando después de la ingesta adecuada de yodo, la excreción de la mediana urinaria de yodo es de 100-199 $\mu \mathrm{g} / 1$ (24). En un estudio reciente sobre valoración de los niveles de Yodo en escolares entre los 8 y 18 años del Quindío como indicadores de desórdenes por deficiencia de Yodo, se mostró que en esa población el déficit general encontrado fue de $28,8 \%$ y los niveles aumentados se encontraron en $43 \%$ de los sujetos estudiados (12).

El hipotiroidismo causado por déficit de yodo dejó de ser una causa importante de morbilidad desde que se inició la yodización de la sal hacia 1950; se considera que el papel del yodo sería permisivo, en ausencia del mismo las alteraciones tiroideas serían las relacionadas con el bocio multinodular; en su presencia, la prevalencia de estas entidades sería menor y mayor la de la ETA (23). Llamó la atención en el presente estudio el excesivo consumo de sal evidenciado al adicionarla a comidas preparadas, a las frutas y al consumo de alimentos salados en paquete.

La importancia de la ETA radica en el incremento de la probabilidad de desarrollar con el tiempo una hipofunción tiroidea sub clínica o manifiesta $(5,16)$, con efectos cardiovasculares dada su repercusión sobre la enfermedad ateroesclerótica y la dislipidemia. 
En el presente estudio se encontró que en total un $63 \%$ de las personas tuvieron uno o más síntomas de los que se presentan en el hipotiroidismo subclínico. Las manifestaciones en orden de frecuencia fueron las relacionadas con el ánimo (depresión, fatiga, somnolencia, astenia y adinamia); así mismo, más de la tercera parte de las personas manifestaron sequedad en la piel, mal color y caída del cabello. En la misma proporción se encontraron anormalidades en el peso siendo la más frecuente el aumento entre 1 y 10 kilogramos en el último año, menos frecuentemente refirieron disminución en el peso. Una cuarta parte de los participantes refirieron edemas frecuentes sobre todo de manos y pies. En menor proporción se encontraron parestesias y disestesias sobre todo en extremidades, intolerancia al frío, estreñimiento y dolores óseos.

En conclusión, se encontró una elevada prevalencia de TSH elevada en personas que previamente no tenían conocimiento de dicha alteración; los niveles elevados de anti- TPO sugieren la necesidad de realizar un seguimiento para identificar precozmente el desarrollo de ETA. Aunque no se hallaron relaciones con la yoduria, los elevados niveles en la mayoría de la población conducen a la imperiosa necesidad de realizar educación preventiva sobre el consumo de sal. Son necesarias futuras investigaciones que permitan justificar la inclusión de un tamizaje de TSH en personas mayores sobre todo en mujeres ya que las publicaciones coinciden en la elevada frecuencia de hipotiroidismo sobre todo en la menopausia.

Dado que la yoduria y los anti- TPO solo se procesaron en participantes con TSH a partir de 5, la muestra final para el análisis de las relaciones se redujo. Los antecedentes de enfermedades relacionadas con la disfunción tiroidea, así como los antecedentes de exámenes con medios de contraste o el consumo de medicamentos fueron muy escasos y no permitieron realizar un análisis para relacionarlos con las alteraciones encontradas. Se requieren nuevos estudios con muestras mayores y la inclusión de participantes con niveles normales y elevados de TSH.

Agradecimientos: Comunidad de los barrios La Adiela y las Américas; empleadas Hospital Universitario San Juan de Dios y Universidad del Quindío; comunidad en general. 


\section{REFERENCIAS}

1. Cano JF, Tomás P. Patología tiroidea. Alteraciones del calcio. En: Martín A, Cano JF. Atención primaria Conceptos, Organización y Práctica clínica. Volumen 2. Madrid: Elsevier España; 2003. p. 1422- 1426.

2. Rojas W. Inmunología. Medellín: Corporación para investigaciones biológicas; 1995. p. 350- 353

3. Marsiglia I. Enfermedad tiroidea asociada a otras enfermedades sistémicas. Gac Méd Caracas. [Internet]. 2005; 113(4): 453-465. Disponible en: http://www.scielo.org.ve/ scielo.php?pid=S0367-47622005000400002\&script=sci_arttext. Consultado: Junio de 2008.

4. Pinzón J. Enfermedades tiroideas autoinmunes. En: Anaya JM, Shoenfeld $Y$, Correa $P$ García- Carrasco P, Cervera R. Autoimunidad y enfermedad autoinmune. Medellín: Corporación para investigaciones biológicas; 2005. p. 331-337.

5. Díaz JJ. Hipotiroidismo en el paciente anciano: importancia clínica y dificultades en el diagnóstico y tratamiento. Med Clin (Barc) 1998; 111: 742-750.

6. Barón G. Prevalencia de hipotiroidismo subclínico en la población post-menopáusica Revista de menopausia. 2001; 7(2):194-198.

7. Reed P, Davies T, Schlumberger MJ, Hay I. Fisiología del tiroides y evaluación diagnóstica en los pacientes con trastornos tiroideos. En: Kronenberg H, Melmed H, Polonsky K, Reed P. Tratado de Endocrinología. Barcelona: Elsevier España; 2009. p. 305334.

8. Soveid M, Monabbati A, Sooratchi L, Dahti S. The effect of iodine prophylaxis on the frequency of thyroiditis and thyroid tumors in Southwest, Iran. Saudi Medical Journal. [Internet] 2007; 28(7):1034-8. Disponible en: http://ipac.kacst.edu.sa/ eDoc/2007/166082_2.pdf Consultado: Junio de 2008.

9. Camargo RY, Tomimori EK, Neves SC, Knobel M, Medeiros-Neto G. Prevalence of chronic autoimmune thyroiditis in the urban area neighboring a petrochemical complex and a control area in Sao Paulo, Brazil. Clinics. 2006; 61(4):307-12.

10. Poncin S, Senou M, Delaigle A, Daumerie Ch. Boschi A, Costagliola S, Boucquey M, Gérard AC, Colin IM, Many MC. Animal Models of Thyroid Autoinmunity: Role of cellular oxidative stress and protective mechanisms. En: Wiersinga WM, Drexhage HA, Weetman AP, Butz S, eds. The thyroid and Autoinmunity, Merck European Symposium; 2006; Noordwijk The Netherlands, June 15-18; 2007. p: 36- 45.

11. Pino S, Fang SL, Braverman LE. Ammonium persulfate: a safe alternative oxidizing reagent for measuring urinary iodine. Clin Chem. 1996; 42(2):239-43.

12. Gallego ML, Loango N, Londoño ÁL y Landázuri P. Niveles de excreción urinaria de yodo en escolares del Quindío, 2006-2007. Rev. salud pública (Bogotá). 2009; 11 (6): 952-960.

13. Iodine status worldwide: WHO Global Database on lodine Deficiency [Internet]. Editors: Bruno de Benoist Maria Andersson, Ines Egli, Bahi Takkouche, Henrietta Allen. WHO, Geneva, 2004. Disponible en: http://whqlibdoc.who.int/ publications/2004/9241592001.pdf. Consultado en junio de 2008.

14. Mayayo E, Ferrández A. Labarta JI. Interpretación de las pruebas tiroideas. An Esp Pediatr. 2002; 56 [Supl 4]: 42-52.

15. Fardela C. Epidemiologia de los trastornos tiroideos en Chile. Boletín de la escuela de medicina; Universidad católica de Chile [Internet]. 2000; 29 (3). Disponible en: http://escuela.med.puc.cl/publ/boletin/Tiroidea/EpidemiologiaTrastornos.html Consultado: Junio de 2008.

16. The National Academy of Clinical Biochemistry. Guía de consenso para el diagnóstico y seguimiento de la enfermedad tiroidea Parte III. Acta Bioquím Clín Latinoam. 2007; 41 (1): 87-119. 
17. Pearce EN, Farwell AP, Braverman LE. Thyroiditis. N engl j med. 2003; 348 (26), p: 2646-2655.

18. Karmisholt J, Laurberg P. Serum TSH and serum thyroid peroxidase antibody fluctuate in parallel and high urinary iodine excretion predicts subsequent thyroid failure in a 1-year study of patients with untreated subclinical hypothyroidism. European Journal of Endocrinology. 2008; 158: 209-215.

19. Walsh JP, Bremner AP, Feddema P, Leedman PJ, Brown SJ, O'Leary P. Thyrotropin and Thyroid Antibodies as Predictors of Hypothyroidism: A 13-Year, Longitudinal Study of a Community-Based Cohort Using Current Immunoassay Techniques. J Clin Endocrinol Metab. 2010; 95(3):1095-1104.

20. Cruz SM, Nicolás M, Ausar SF, Silvera ER. Prevalencia de anticuerpos antitiroglobulina y antimicrosomales en enfermedad tiroidea autoinmune. Arch. argent. alerg. inmunol. clin. 1999; 30 (4): 22-9.

21. Toni M, Anda E, Pineda J, Martínez de Esteban JP, Ollero. Trastornos tiroideos por amiodarona. An. Sist. Sanit. Navar. 2009; 32 (3): 363-370.

22. Calé J, Lutfi RJ. El litio y su influencia en la función tiroidea. Revista Argentina de Endocrinología y Metabolismo. 2009; 43 (3): 37-42.

23. Girón JA, Arizcorreta A, Brun $F$.Enfermedad tiroidea de patogenia inmunológica. Medicine. 2000; 8 (17): 903-9.

24. Organización Panamericana de la Salud, Organización Mundial de la salud [Internet]. Reducción del consumo de sal en la población. Foro de la OMS sobre la Reducción del Consumo de Sal en la Población; 2006; París Francia Oct 5 al 7; 2008; p:9-22 Disponible en: http://www.who.int/dietphysicalactivity/salt-report-SP.pdf Consultado: Septiembre de 2010. 